Afrika Focus, Vol. 14, Nr. 1, 1998, pp. 43-54.

\title{
DEFINITION DE L'ADJECTIF EN LANGUES BANTU
}

\author{
Jean BAKA \\ Université Libre de Bruxelles \\ Section de Linguistique \\ Musée Royal de l'Afrique Centrale \\ Leuvensesteenweg 13 \\ B-3080 Tervuren
}

\section{SUMMARY}

\section{DEFINITION OF THE ADJECTIVE IN BANTU LANGUAGES}

In Bantu, as in other language groups, the definition of the adjective depends on the type of criteria that one uses. Some authors make use of morphological criteria, some of syntactic criteria and others of semantic criteria. From this variety of criteria there follows a diversity of definitions. One can reach, nevertheless, a more appropriate definition, by having recourse to the whole set of possible criteria but giving primacy to the syntactic ones. Such definition allows us - on the one hand - to bypass the dichotomy between nouns and pronouns which is founded on a purely morphological criterion " the form of the prefix " and - on the other hand - to restrict the meaning of the word 'adjective' to a purely semantic notion. 
KEY WORDS : linguistics, Bantu, adjective

\section{INTRODUCTION}

Quand l'on parcourt les dictionnaires spécialisés ou les dictionnaires de langues ou encore les grammaires, on est amené à penser que la notion d'adjectif est clairement établie et que son identification et sa définition ne posent aucun problème. Pourtant quand l'on examine les critères de définition ou de différenciation qui sont donnés pour justifier la distinction entre les substantifs et les adjectifs, on constate malheureusement que ceux-ci sont souvent inopérants; on conçoit ici l'adjectif comme l'ensemble qui regroupe les notions traditionnelles d'adjectifs qualificatifs et d'adjectifs déterminatifs (MAROUZEAU, 1951). En français et en anglais, on inclut dans les adjectifs déterminatifs, les numéraux, les démonstratifs, les indéfinis, les possessifs, les interrogatifs et les relatifs (GLEASON, 1955; GREVISSE \& GOOSSE, 1990; LYONS, 1995).

Tout en passant en revue ces critères, le but de cet article est de proposer une approche qui permettrait l'identification et la définition sans ambiguïté de l'adjectif dans les langues bantu connues, voire dans les langues européennes comme le français ou l'anglais.

\section{CRITERES DE DEFINITION}

1.1. Pour définir l'adjectif (Adj), on note en bantu trois tendances majeures dans les travaux de type descriptif, théorique ou comparatif.

La première tendance, qui fait autorité dans les travaux actuels, est inspirée de l'oeuvre de MEEUSSEN (1967).

Elle identifie l'adjectif sur la base de trois critères qui sont:

$1^{\circ}$ la forme du préfixe (PN ou PP),

$2^{\circ}$ le caractère omniclasse,

$3^{\circ}$ la dépendance syntaxique (STAPPERS, 1965:177; MEEUSSEN, 1967:104).

Aussi, STAPPERS (sd:50) estime que: le cas le plus courant dans les langues bantu est que l'adjectif est une forme nominale, dépendante du substantif (Sbst) avec lequel il s'accorde en classe. 
En clair le substantif est composé d'un préfixe propre (PN) qui varie généralement en singulier et pluriel, l'adjectif lui est composé d'un thème et d'un préfixe qui est dicté par le substantif (PA) dont il dépend.

Les exemples suivants repris au swahili (G), langue bantu d'Afrique de l'est et de la République Démocratique du Congo, paraissent confirmer cette définition puisqu'on relève une identité formelle entre le PN et le PA.

(1)

$\begin{array}{clll}\text { Cl. } & \text { Sbst. } & \text { Adj. } & \text { Traduction } \\ 1 & \text { m-tu } & \text { m-nene } & \text { "homme corpulent" } \\ 2 & \text { wa-tu } & \text { wa-nene } & \text { "hommes corpulents" } \\ 3 & \text { m-to } & \begin{array}{l}\text { m-pana } \\ \text { mi-refu }\end{array} & \text { "large rivière" } \\ 4 & \text { mi-nazi } & \text { "cocotiers élevés" } \\ 6 & \text { ma-kombe } & \text { ma-pya } & \text { "plats neufs" } \\ 9 & \text { N-doo } & \text { N-pya } & \text { "seau neuf" } \\ 10 & \text { N-doo } & \text { N-pya } & \text { "seaux neufs" }\end{array}$

Les adjectifs de MEEUSSEN se résument à l'adjectif qualificatif puisque les numéraux (Num), les démonstratifs (Dém), les indéfinis (Ind), les interrogatifs (Int) et les possessifs (Pos) dont la forme du préfixe est toujours, semble t-il, pronominale (PP) n'en font pas partie (MEEUSSEN, 1967:97). Dans les faits, on constate cependant que cette tendance occulte la situation de certains déterminants tels les numéraux qui apparaissent dans nombre de parlers bantu, comme on peut le noter ci-après à partir des données du swahili, avec des PA formellement identiques aux PN des substantifs.

(2)

\begin{tabular}{|c|c|c|c|c|}
\hline $\begin{array}{l}\mathrm{Cl} . \\
1\end{array}$ & $\begin{array}{l}\text { Sbst. } \\
\text { m-tu } \\
\text { homme }\end{array}$ & $\begin{array}{l}\text { "Adj. " } \\
\text { m-fupi } \\
\text { Court }\end{array}$ & $\begin{array}{l}\text { Num. } \\
\text { m-moja } \\
\text { un }\end{array}$ & $\begin{array}{l}\text { Dém. } \\
\text { yu-le } \\
\text { ce là-bas }\end{array}$ \\
\hline 3 & $\begin{array}{l}\text { m-ti } \\
\text { arbre }\end{array}$ & $\begin{array}{l}\text { m-zuri } \\
\text { Beau }\end{array}$ & $\begin{array}{l}\text { m-moja } \\
\text { un }\end{array}$ & $\begin{array}{l}\text { u-le } \\
\text { ce là-bas }\end{array}$ \\
\hline 4 & $\begin{array}{l}\text { mi-ti } \\
\text { arbres }\end{array}$ & $\begin{array}{l}\text { mi-zuri } \\
\text { Beaux }\end{array}$ & $\begin{array}{l}\text { mi-wili } \\
\text { deux }\end{array}$ & $\begin{array}{l}\text { i-le } \\
\text { ces là-bas }\end{array}$ \\
\hline 9 & $\begin{array}{l}\mathbf{N} \text {-umba } \\
\text { maison }\end{array}$ & $\begin{array}{l}\text { N-defu } \\
\text { Long }\end{array}$ & $\begin{array}{l}\mathrm{N} \text {-moja } \\
\text { un }\end{array}$ & $\begin{array}{l}\text { yi-le } \\
\text { ce là-bas }\end{array}$ \\
\hline
\end{tabular}




$\begin{array}{llll}\mathbf{N} \text {-umba } & \text { N-defu } & \text { N-bili } & \text { zi-le } \\ \text { maisons } & \text { Longs } & \text { deux } & \text { ces là-bas }\end{array}$

La seconde tendance - que l'on pourrait attribuer à GUTHRIE (1953; 1967-71) ne semble pas considérer comme critère primordial la forme du préfixe. Ici l'adjectif est défini comme une forme dépendante omniclasse (DOKE, 1943:33; GUTHRIE, 1953:10; 1967:13).

Définissant l'adjectif après avoir examiné et comparé les deux tendances, MPIRANYA (1996:158) souligne qu'en langue bantu, l'on peut dire que l'accord des adjectifs se fait en classe et en nombre parce que chaque classe porte en elle son nombre. Il est à remarquer que c'est le schème d'accord qui compte le plus, plutôt que la forme du préfixe de dépendance (PA), car elle peut être différente $(d u P N)$ dans certaines classes.

L'examen des données qu'on a récoltées dans près de 300 langues et parlers bantu confirme cette assertion (BAKA, 1998).

En effet, dans une grande partie de langues et parlers bantu étudiés, la forme du préfixe d'accord qui intervient dans l'adjectif peut être différente du préfixe qu'utilise le substantif:

* à certaines classes tel qu'on peut le noter en ruund $(\mathrm{K})$, parler bantu de la République Démocratique du Congo, où le PA est formellement identique au $\mathrm{PN}$ en cl. 1 mais différent de celui-ci aux cl. 3 et 4 :

(3)

$\begin{array}{llll}1 & \text { mu-ntw } & \text { mu-ymp } & \text { "le mauvais homme" } \\ 3 & \text { mu-tònd } & \text { wu-lêmp } & \text { "un arbre long" } \\ 4 & \text { mi-tònd } & \text { yi-lêmp } & \text { "des arbres longs" }\end{array}$


* à l'ensemble des classes comme ci-après en vili $(\mathrm{H})$, parler koongo du Congo-Brazzaville et du Gabon, où quelque soit le déterminant considéré le PA est formellement identique au PP et (donc) différent du PN :

(4)

$\begin{array}{llllll}\begin{array}{l}\text { Cl. } \\ 1\end{array} & \begin{array}{l}\text { Subst. } \\ \text { m-tu } \\ \text { homme }\end{array} & \begin{array}{l}\text { «Adj. » } \\ \text { ú-nene } \\ \text { Grand }\end{array} & \begin{array}{l}\text { Num. } \\ \text { ú-mosi } \\ \text { un }\end{array} & \begin{array}{l}\text { Dém. } \\ \text { w-úna } \\ \text { ce là-bas }\end{array} & \begin{array}{l}\text { Pos. } \\ \text { w-á:mi } \\ \text { à moi }\end{array} \\ 3 & \begin{array}{l}\text { mw-á:nza } \\ \text { toit }\end{array} & \begin{array}{l}\text { ú-nene } \\ \text { Grand }\end{array} & \begin{array}{l}\text { ú-mosi } \\ \text { un }\end{array} & \begin{array}{l}\text { w-úna } \\ \text { ce là-bas }\end{array} & \begin{array}{l}\text { w-á:mi moi } \\ \text { à moi }\end{array} \\ 9 & \begin{array}{l}\text { N-vúmbu } \\ \text { hippopotame }\end{array} & \begin{array}{l}\text { í-nene } \\ \text { Gros }\end{array} & \begin{array}{l}\text { ímosi } \\ \text { un }\end{array} & \begin{array}{l}\text { y- ína } \\ \text { ce là-bas }\end{array} & \begin{array}{l}\text { y-á:mi } \\ \text { à moi }\end{array}\end{array}$

Le passage des voyelles $\mathbf{u}$ et $\mathbf{i}$ respectivement à $\mathbf{w}$ et $\mathbf{y}$ s'explique en bantu par des règles de contact vocalique.

Dans d'autres langues assez représentatives du bantu, on note un phénomène d'alternance (libre) de la forme du PA, celle-ci peut être identique au PN ou différente de lui comme en témoignent les données suivantes recueillies en sena (N), langue bantu du Mozambique (BAKA : 1998) :

(5)

$\begin{array}{llll} & \text { Sbst. } & \text { Adj } & \\ 4 & \text { mi-ti } & \text { mi-kulu } & \text { "grands } \\ 4 & \text { mi-ti } & \text { i-kulu } & \text { "grands arbres" }\end{array}$

Ainsi, si dans la hiérarchie des critères d'identification et de définition des adjectifs, on place en premier lieu la forme nominale du préfixe dépendant ou d'accord (MEEUSSEN, 1967; LAFON, 1997), on est amené à conclure qu'en 
vili, il n'existe pas d'adjectif - ce qui est contraire aux conclusions auxquelles a abouties BOUKA (1994) - tandis qu'en ruund l'adjectif existerait en cl.1 mais non aux cl.3 et 4 . Et que dira-t-on du sena qui, à la même classe, atteste deux formes de PA?

La troisième tendance, que l'on pourrait qualifier d'eurocentriste, est résumée en ces termes par WELMERS (1973:249) qui du reste n'y adhère pas totalement : The term 'adjective' may be applied to any form which is reflected by an English adjective in translation, without reference to its derivation or grammatical function in the language being described.

Or, comparant la liste des adjectifs du sotho-nord (S) - le sotho est une langue bantu de la République Sud-Africaine - avec celle des adjectifs de l'anglais, POULOS \& LOUWRENS (1994:93) notent que:

It is clear from the above list of adjectival stems that not all adjectives as they are known in English, are classified as adjectives in Northern Sotho. In fact, certain adjectives as we know them in English, such as, for example, 'strong', 'angry', 'dry', etc. are expressed by some of the other qualificatives in Northern Sotho. So it is important to remember that there isn't a one-to-one correspondence between the adjectives in English (or any other language for the matter) and those of Northern Sotho.

Les exemples suivants recueillis en sotho-nord montrent en effet qu'aux adjectifs "clever" et "fierce" de l'anglais correspondent les substantifs de cl.14 buhlali et bugali qui se traduisent respectivement "cleverness" et "fierceness" :

$\begin{array}{llll}\text { Sbst.1 } & \text { Con. } & \text { Stbt. 2 } & \\ \text { Ngw-ana } & \text { yo } & \text { bu-hlali } & \text { "a clever child" } \\ \text { Child } & \text { of } & \text { cleverness } & \text { "fierce dogs" } \\ \text { Dim-psa } & \text { tse } & \text { bu-gali } & \\ \text { Dogs } & \text { of } & \text { fierceness } & \end{array}$

1.2. Pour ce qui est des langues occidentales comme le français ou l'anglais, les définitions de l'adjectif que l'on rencontre dans les dictionnaires ou les grammaires sont conformes, du moins en théorie, à l'étymologie du mot en grec 
epítheton, "ce qui est ajouté ou joint" (MAROUZEAU, 1951; BUSSMANN, 1995). On peut par exemples citer les quelques définitions qui suivent:

L'adjectif est le mot qui est joint au nom pour exprimer la qualité de l'objet ou de l'être, ou de la notion désignée par ce nom (adjectif qualificatif) ou bien encore permettre à ce nom d'être actualisé dans une phrase (adjectif déterminatif) (DUBOIS \& al, 1973).

Pour l'anglais,

adjective is a word that indicates a quality of the person or thing referred to by a noun (OXFORD DICTIONARY, 1991).

\title{
A beautiful smile \\ Beautiful smiles \\ The house was quiet
}

Pour le français,

l'adjectif est un mot qui varie en genre et en nombre, genre et nombre qu'il reçoit, par le phénomène de l'accord du nom auquel il se rapporte. Il est apte à servir d'épithète ou d'attribut. Du point de vue sémantique, l'adjectif exprime une manière d'être, une qualité de l'être ou de la chose désignées par le nom auquel il se rapporte (GREVISSE \& GOOSSE, 1990).

(8)

\author{
Un petit bonhomme \\ Ils sont gentils \\ Les vaches blanches et noires
}

On relève qu'en anglais où les adjectifs dits qualificatifs sont invariables, la définition de l'adjectif s'appuie sur des critères sémantiques tandis qu'en français où les adjectifs seraient toujours variables, celle-ci s'appuie sur des critères morphologiques et syntaxiques. Il existe toutefois en anglais des critères de type morphologique (notamment la flexion en - $\partial \mathrm{r}$ et en -ist) pour identifier les adjectifs mais ceux-ci sont malheureusment inopérants à une grande échelle (GLEASON, 1955:96-97). 
Pour s'en tenir uniquement au français, on constate, quand l'on parcourt la Nouvelle Grammaire du Français de GREVISSE \& GOOSSE,

* d'une part que certains adjectifs peuvent être invariables comme on peut le relever ci-après:

(9)

\author{
Des yeux bleu clair \\ Le pavement est de carreaux gros bleu \\ Ces étoffes coûtent cher
}

* et que d'autre part, certaines formations que ces deux auteurs présentent comme des adjectifs occasionnels sont en réalité des substantifs.

Des rubans cerise
Des vestes ventre de biche
Des fauteuils empire
Des airs bonhomme

L'invariabilité possible de l'adjectif dans certains cas, l'existence de noms employés adjectivement posent le problème de la frontière qui peut être établie entre le substantif et l'adjectif français. En se référant aux exemples en 10, on constate que les tests distributionnels (on identifie les unités de la langue à partir des différentes positions qu'elles occupent dans l'énoncé) qui sont mis en avant par la grammaire traditionnelle du français pour justifier la séparation entre le substantif et l'adjectif s'avèrent inopérants (CREISSELS, 1991).

1.3. En théorie générale, BHAT (1994) a dressé les carastéristiques sémantique, fonctionnelle et morphosyntaxique principales qui, semble t-il, permettraient d'identifier et de différencier les catégories syntaxiques majeures dans n'importe quelle langue du monde. On les énumère ci-après telles qu'elles apparaissent dans le travail de l'auteur (BHAT, 1994:18-21). 
Noun

Adjective

Verb

Adverb
Identifying a participant

Suggesting a cluster of proprieties

Emphasizing the possessor of properties

Denoting objects

Independent as a head noun

Modifying a noun

Denoting a single property

Emphasizing the property itself

Denoting property concepts

Dependent on head noun

Predicating an event

Characterizing the referents of the arguments

Denoting changing characteristics

Independent as the nucleus in a sentence

Modifying a verb

Free from the head (nucleus)

On peut noter qu'à l'exception de celles qui sont soulignées, ces caractéristiques sont toutes d'ordre sémantique. Or, on vient déjà de le souligner, l'identification des adjectifs à partir des seules caractéristiques sémantiques est dangereuse et peu pratique.

\section{NOUVELLES CONSIDERATIONS SUR L'ADJECTIF}

La lecture de l'ensemble des données permet de relever que la variabilité en nombre et/ou en genre bien qu'elle soit présente en bantu, en français (ou en allemand) n'est pas générale dans les langues du monde. Le sens que porte un lexème ne suffit pas non plus à déterminer la catégorie à laquelle il appartient. C'est plutôt la syntaxe qui intervient de manière déterminante dans 
l'identification et la différenciation des catégories grammaticales.

Aussi, pour définir l'adjectif il semble plus aisé d'accorder la primauté à des critères d'ordre syntaxique. Les critères d'ordre sémantique et morphologique ne doivent cependant pas être évacués. En y recourant, il y a moyen de préciser par des nuances supplémentaires la définition retenue. A partir des exemples suivants du français :

\author{
le pull-over rouge \\ le pull-over \\ le rouge \\ le rouge est mis
}

ou ceux ci-après du tswana (S), langue bantu parlée au Botswana

on constate que le substantif peut constituer à lui seul un syntagme nominal; toutefois, l'adjectif ne peut le faire que s'il renvoie à un référent qui a été effacé par une opération de réduction. La théorie "guillaumienne" de l'incidence explique cela par le fait que le support de l'adjectif est extérieur à son apport alors que celui du substantif ne sort pas du champ que couvre son apport, c'està-dire que l'apport de significations du substantif annonce la nature du support, celui de l'adjectif n'annonce pas la nature du support (TABI-MANGA, 1992). Or, ce procédé de réduction existe dans des langues qui appartiennent à des familles très éloignées. 


\section{EN GUISE DE CONCLUSION}

En définitive, on considérera l'adjectif comme toute forme dépendante non verbale qui ne peut constituer à elle seule un syntagme qu'en faisant référence à un substantif ou à un autre syntagme qui a été effacé à la suite d'une opération de réduction.

Pour revenir au bantu, le mérite de la seconde tendance réside dans le fait qu'elle néglige la dichotomie noms versus pronoms établie par la première tendance et qui est fondée sur la seule forme du préfixe, en ce sens qu'est défini comme nom "toute forme qui a un PN" et comme pronom "toute forme qui porte un PP".

A la différence des autres déterminants du nom, le préfixe des substantifs de cl.14 ou de toute autre classe - que LOUWRENS (1994) appelle « qualificative nouns " et MPIRANYA (1996) adjectifs de discours - ne dépend pas du déterminé; à moins de réduire la notion d'adjectif établie par la grammaire traditionnelle dans les langues indo-européennes à une simple classe sémantique (comme le font les tenants de la troisième tendance), les déterminants dont le préfixe ne dépend pas du déterminé ou mieux du substantif ne peuvent être considérés comme des adjectifs. C'est pourquoi il y a lieu de distinguer - en bantu et dans les autres langues du monde où on peut établir une classe d'adjectifs - les déterminants dépendants (adjectifs qualificatifs et adjectifs déterminatifs) d'une part et les déterminants non dépendants (substantifs qui qualifient ou déterminent) d'autre part. Les premiers relèvent de la syntaxe, les seconds de la sémantique.

\section{REFERENCES}

BAKA, J., L'adjectif en bantu, PhD en cours, ULB, 1998.

BHATD, N.S., The Adjectival Category, Amsterdam, 1994.

BOUKA, L.Y., L'accord des adjectifs du protobantou en zone H. In : Africana Linguistica XI, 1994.

BUSSMANN, H., Dictionary of Language and Linguistics, London, 1995. 
DOKE, C.M., Outline Grammar of Bantu, Johannesburg, 1943.

DUBOIS, J. \& al., Dictionnaire de linguistique, Paris, 1973.

CREISSELS, D., Description des langues négro-africaines et théorie syntaxique, Grenoble, 1991.

GLEASON, H.A., An Introduction to Descriptive Linguistics, New York, 1955.

GREVISSE, M., \& GOOSSE, Nouvelle grammaire française, Paris, 1990.

GUTHRIE, M., The Bantu Languages of Western Equatorail Africa, London, 1953. Comparative Bantu, vol.1-4, London, 1967-71.

LAFON, M., L'expression de la qualité en shingazidja, l'adjectif. In:_Linguistique Africaine, 1997.

LOUWRENS, L.J., Dictionary of Northen Sotho: Grammatical Terms. Pretoria, 1994.

LYONS, J., Language and Linguistics. An Introduction, New York, 1995.

MAROUZEAU, J., Lexique de la terminologie linguistique, Paris, 1951.

MARTINET, A., La Linguistique. Guide alphabétique, Paris, 1969.

MEEUSSEN, A.E., Bantu Grammatical Reconstructions, Tervuren, 1967.

MPIRANYA, F., Perspective fonctionnelle en linguistique comparée des langues bantu, doctorat, Lyon-3, 1996.

POULOS, \& LOUWRENS, A Linguistic Analysis of Northen Sotho, Pretoria, 1994.

STAPPERS, L., Introduction à la linguistique africaine (manuscrit), sd., Tervuren.

STAPPERS, L., Het Hoofdtelwoord in de Bantoe-Talen. In: Africana Linguistica II, 1965.

TABI-MANGA, J., De la grammaire de l'ewondo à une théorie du mot, Paris, 1992.

WELMERS, WM.E., African Language Structures, Berkeley, 1973. 